\title{
OSCILLATORY BEHAVIOR OF ORTHOGONAL POLYNOMIALS
}

\author{
ATTILA MÁTE $E^{1}$, PAUL NEVAI ${ }^{2}$ AND VILMOS TOTIK ${ }^{3}$
}

\begin{abstract}
Let $d \alpha$ be a positive Borel measure in $[-1,1]$ with $\alpha^{\prime}>0$ a.e. It is shown that the polynomials $p_{n}$ orthonormal with respect to this measure oscillate almost everywhere in $[-1,1]$. A function $F$ is also described that is a pointwise bound for $p_{n}$, exceeded only on sets of small measure. It is shown that $F$ is the best possible.
\end{abstract}

1. Introduction and statement of results. Let $\alpha$ be a nondecreasing function on $[-1,1]$ with infinitely many points of increase, and denote by $p_{n}(x)=p_{n}(d \alpha, x)$ the polynomials orthonormal with respect to $d \alpha$; that is, $p_{n}$ is a polynomial of degree $n$ with positive leading coefficient $\gamma_{n}=\gamma_{n}(d \alpha)$ such that

$$
\int_{-1}^{1} p_{m}(x) p_{n}(x) d \alpha(x)=\delta_{m n} \quad(m, n \geqslant 0) .
$$

The usual pointwise asymptotic formulas for orthogonal polynomials on a finite interval have the form

$$
p_{n}(x) \cong A(x) \sin \left(k_{n}(x)+B(x)\right),
$$

and the oscillatory behavior of the sequence $\left\{p_{n}(x)\right\}$ can easily be deduced from this expression. However, all of the results concerning pointwise asymptotics are rather special, and the asymptotic formulas for classical polynomials and for the Pollaczek polynomials (see e.g. [14, Chapter 8 and Appendix]) indicate that there is very little hope for obtaining pointwise asymptotics without imposing some conditions on the measure. Nevertheless, the oscillatory behavior of the sequence $\left\{p_{n}(x)\right\}$ can be proved for a fairly general class of orthogonal polynomials. In fact, we have

THEOREM 1. Assume $\alpha^{\prime}>0$ almost everywhere in $[-1,1]$. Then, for almost every $x \in[-1,1]$, the set of accumulation points of the sequence $\left\{p_{n}(x)\right\}_{n=0}^{\infty}$ is an interval $I(x)$ symmetric about the origin such that its length

$$
|I(x)| \geqslant 2 \sqrt{2 / \pi}\left(\alpha^{\prime}(x)\right)^{-1 / 2}\left(1-x^{2}\right)^{-1 / 4} .
$$

Received by the editors February 19, 1985.

1980 Mathematics Subject Classification. Primary 42C05.

Key words and phrases. Orthogonal polynomials, Szegö's theory, weak convergence, mean convergence.

${ }^{1}$ Supported by the National Science Foundation under Grant No. DMS- 8400906 and by the PSC-CUNY Research Award Program of the City University of New York under Grant No. 664252. Work partially completed at the Institute for Advanced Study during the summer of 1984.

${ }^{2}$ Supported by the National Science Foundation under Grant No. MCS- 8300882 .

${ }^{3}$ Work completed while visiting Ohio State University. 
Hence,

$$
\limsup _{n \rightarrow \infty} p_{n}(x) \geqslant \sqrt{\frac{2}{\pi}}\left(\alpha^{\prime}(x)\right)^{-1 / 2}\left(1-x^{2}\right)^{-1 / 4}
$$

and

$$
\liminf _{n \rightarrow \infty} p_{n}(x) \leqslant-\sqrt{\frac{2}{\pi}}\left(\alpha^{\prime}(x)\right)^{-1 / 2}\left(1-x^{2}\right)^{-1 / 4}
$$

almost everywhere in $[-1,1]$.

COROLLARY. Let $\phi$ be a function assuming positive finite values almost everywhere in $[-1,1]$, and suppose that

$$
\limsup _{n \rightarrow \infty}\left|p_{n}(x)\right| \leqslant \phi(x)
$$

holds for $-1 \leqslant x \leqslant 1$ and $n=0,1,2, \ldots$ Then we have

$$
\alpha^{\prime}(x) \geqslant 2 /\left(\pi \phi^{2}(x) \sqrt{1-x^{2}}\right)
$$

for almost every $x \in[-1,1]$.

REMARKS. (i) For the classical orthonormal polynomials we have equality in (1), (2), and (3). This shows that the constant $2 / \pi$ in (5) is the best possible (cf. [8, Theorem 7.5, p. 128; 2, formula (VII.7), p. 80]).

(ii) Theorem 1 need not hold for every $x \in[-1,1]$; e.g., the orthonormal Chebyshev polynomials take only the values $\{0, \pm \sqrt{2 / \pi}\}$ at $x=0$.

(iii) Under the assumptions of Theorem 1 there is no oscillation outside $[-1,1]$. Indeed, these assumptions ensure that if $z$ is a complex number not belonging to $[-1,1]$ then

$$
p_{n+1}(z) / p_{n}(z) \rightarrow z+\sqrt{z^{2}-1}
$$

where that branch of the square root is taken for which $\left|z+\sqrt{z^{2}-1}\right|>1$ (see [11, Remark 3, formula (26), p. 211; 12, Theorem 2, p. 105]; cf. also [4], where an error of [11] is pointed out-this error is corrected in [12]; in [5], a substantially simpler proof of the quoted result of [11 and 12] is given). Thus, for every complex $z \notin[-1,1]$ we have

$$
\lim _{n \rightarrow \infty}\left|p_{n}(z)\right|=\infty \text {. }
$$

(iv) The assumption that $\alpha^{\prime}(x)>0$ a.e. in $[-1,1]$ is needed in Theorem 1 . Indeed, if $\alpha^{\prime}$ vanishes outside a proper subinterval $[a, b]$ of $[-1,1]$, then by a linear transformation we can map $[a, b]$ onto $[-1,1]$. The preceding remark then shows that $\lim _{n \rightarrow \infty}\left|p_{n}(x)\right|=\infty$ holds for every $x \notin[a, b]$.

Our next result says that, in spite of (2) and (3), the sequence $\left\{\left|p_{n}(x)\right|\right\}$ essentially remains under the (absolute value of the) right sides of these formulas (we write $|E|$ for the Lebesgue measure of the set $E$ ).

THEOREM 2. Assume $\alpha^{\prime}>0$ almost everywhere in $[-1,1]$, and let $\varepsilon, 0<\varepsilon<1$, be arbitrary. Then we have

$$
\lim _{n \rightarrow \infty}\left|\left\{x:\left|p_{n}(x)\right|>(1+\varepsilon) \sqrt{\frac{2}{\pi}}\left(\alpha^{\prime}(x)\right)^{-1 / 2}\left(1-x^{2}\right)^{-1 / 4}\right\}\right|=0 .
$$


Moreover, for every Lebesgue measurable set $E \subset[-1,1]$, we have

$$
\begin{gathered}
\limsup _{n \rightarrow \infty}\left|\left\{x \in E: p_{n}(x)>(1-\varepsilon) \sqrt{\frac{2}{\pi}}\left(\alpha^{\prime}(x)\right)^{-1 / 2}\left(1-x^{2}\right)^{-1 / 4}\right\}\right| \\
\geqslant(1 / \pi)|E| \operatorname{arc} \cos (1-\varepsilon)
\end{gathered}
$$

and

$$
\begin{gathered}
\limsup _{n \rightarrow \infty}\left|\left\{x \in E: p_{n}(x)<-(1-\varepsilon) \sqrt{\frac{2}{\pi}}\left(\alpha^{\prime}(x)\right)^{-1 / 2}\left(1-x^{2}\right)^{-1 / 4}\right\}\right| \\
\geqslant(1 / \pi)|E| \operatorname{arc} \cos (1-\varepsilon) .
\end{gathered}
$$

For the classical orthogonal polynomials we have equality in (7) and (8); that is, the constant on the right side cannot be improved (cf. [14, Chapter 8]).

2. Proofs. The proofs of the above results depend on the following

Lemma. Assume $\alpha^{\prime}>0$ almost everywhere in $[-1,1]$. Write

$$
e^{i \theta}=x+i y \quad(0 \leqslant \theta \leqslant \pi),
$$

and define $\psi_{n}$ by

$$
\psi_{n}(\theta)=p_{n}(x)-e^{i \theta} p_{n-1}(x) .
$$

Let $F$ be a subset of the interval $(0, \pi)$, and let $S$ be an infinite set of positive integers such that, for each $\theta \in F$,

$$
\sup \left\{\left|\psi_{n}(\theta)\right|: n \in S\right\}<\infty .
$$

Then there is an increasing sequence $\left\{n_{m}\right\}_{m=1}^{\infty}$ of elements of $S$ such that the relation

$$
\begin{aligned}
y p_{n_{m}+k}(x)=\left|\psi_{n_{m}}(\theta)\right| \sin \left((k+1) \theta-\arg \psi_{n_{m}}(\theta)\right)+o_{\theta}(1) \\
(\theta \in F, 0 \leqslant k \leqslant m, m \rightarrow \infty)
\end{aligned}
$$

holds, where $o_{\theta}(1)$ is a function of $\theta, k$, and $m$ such that $\lim _{m \rightarrow \infty} o_{\theta}(1)=0$ uniformly in $k$ (but not necessarily uniformly in $\theta$ ).

Proof. Consider the recurrence formula

$$
x p_{n}(x)=a_{n+1} p_{n+1}(x)+b_{n} p_{n}(x)+a_{n} p_{n-1}(x)
$$

for $p_{n}$ (cf. e.g. Freud [1, (1.2.4), p. 17]), and write

$$
c_{n}=\left|1-2 a_{n}\right|+2\left|b_{n}\right|+\left|1-2 a_{n-1}\right| \text {. }
$$

By the assumption that $\alpha^{\prime}>0$ a.e. in $[-1,1]$, it follows from the results of Rahmanov [11, p. 212, just before Remark 4] and [12] (which corrects the error made in [11] and pointed out in Máté and Nevai [4]) that

$$
\lim _{n \rightarrow \infty} c_{n}=0
$$

(cf. Máté-Nevai-Totik [6, formula (9.11)] for more details and [5] for a simplified proof of Rahmanov's quoted results).

From the recurrence formula (13) it is easy to see that for the function $\psi_{n}$ defined in (10) we have

$$
\left|\psi_{n+1}(\theta)-e^{i \theta} \psi_{n}(\theta)\right| \leqslant c_{n+1} \sum_{j=n-1}^{n+1}\left|p_{j}(x)\right|
$$


(this inequality occurs in [10, p. 36], where its derivation is given in detail; see also [9]). To estimate the right side here notice that by $(10)$ we have

$$
\left|p_{n-1}(x)\right| \sin \theta \leqslant\left|\operatorname{Im} \psi_{n}(\theta)\right| \leqslant\left|\psi_{n}(\theta)\right|
$$

and

$$
\left|p_{n}(x)\right| \sin \theta \leqslant\left|\operatorname{Im}\left(e^{-i \theta} \psi_{n}(\theta)\right)\right| \leqslant\left|\psi_{n}(\theta)\right| ;
$$

moreover, by noting that $|x|=|\cos \theta| \leqslant 1$, from (13) and (14) we obtain

$$
\left|p_{n+1}(x)\right| \leqslant 3\left(\left|p_{n}(x)\right|+\left|p_{n-1}(x)\right|\right)
$$

for large enough $n$, say $n \geqslant n_{0}$. Thus (15) implies

$$
\left|\psi_{n+1}(\theta)-e^{-i \theta} \psi_{n}(\theta)\right| \leqslant 8 c_{n+1}\left|\psi_{n}(\theta)\right| / \sin \theta \quad\left(n \geqslant n_{0}\right)
$$

(note that $\sin \theta \neq 0$ for $\theta \in F$ since $F \subset(0, \pi)$ ) and so

$$
\left|\psi_{n+1}(\theta)\right| \leqslant\left|\psi_{n}(\theta)\right| \exp \left(8 c_{n+1} / \sin \theta\right) \quad\left(n \geqslant n_{0}\right) .
$$

Iterating this inequality, we obtain

$$
\left|\psi_{n+k}(\theta)\right| \leqslant\left|\psi_{n}(\theta)\right| \exp \left(8 \sum_{j=1}^{k} \frac{c_{n+j}}{\sin \theta}\right) \quad\left(n \geqslant n_{0}, k \geqslant 0\right) .
$$

Now take an increasing sequence $\left\{n_{m}\right\}_{m=1}^{\infty}$ of elements of $S$ with $n_{1}>n_{0}$ such that

$$
\sum_{j=1}^{m} c_{n_{m}+j}<\frac{1}{m} \quad(m=1,2, \ldots)
$$

this inequality can be made to hold in view of (14). By (11), (20), and (21),

$$
\left|\psi_{n_{m}+j}(\theta)\right| \leqslant c(\theta) \quad(m=1,2, \ldots, 0 \leqslant j \leqslant m)
$$

holds for $\theta \in F$ with some $c(\theta)$, and so (19) gives

$$
\begin{aligned}
\left|e^{i\left(n_{m}+j\right) \theta} \psi_{n_{m}+j}(\theta)-e^{i\left(n_{m}+j-1\right) \theta} \psi_{n_{m}+j-1}(\theta)\right| & \leqslant 8 c_{n_{m}+j} c(\theta) / \sin \theta \\
(\theta & \in F, m=1,2, \ldots, 1 \leqslant j \leqslant m) .
\end{aligned}
$$

Given $k \geqslant 0$ and $m \geqslant k$, by adding these inequalities for $j=1,2, \ldots, k$ and taking (21) into account, we obtain

$$
e^{i(n+k) \theta} \psi_{n+k}(\theta)=e^{i n \theta} \psi_{n}(\theta)+o_{\theta}(1) \quad(\theta \in F)
$$

i.e.,

$$
e^{-i \theta} \psi_{n+k}(\theta)=e^{-i(k+1)} \psi_{n}(\theta)+o_{\theta}(\theta) \quad(\theta \in F) .
$$

(12) follows from here by taking imaginary parts (cf. (10) as well). The proof of the Lemma is complete.

Proof of Theorem 1. Writing $I(x)$ for the set of accumulation points of the sequence $\left\{p_{n}(x)\right\}_{n=0}^{\infty}$ and using the notation introduced in (9) and (10), we will first verify the following

Claim. Let $\theta \in[0, \pi]$ be such that $\theta / 2 \pi$ is irrational, and assume that $t$ is an accumulation point of the sequence $\left\{\left|\psi_{n}(\theta)\right|\right\}_{n=1}^{\infty}$. Then

$$
[-t / y, t / y] \subset I(x) \text {. }
$$


To verify this Claim, choose an infinite set $S$ such that

$$
\lim _{n \rightarrow \infty, n \in S}\left|\psi_{n}(\theta)\right|=t .
$$

Then, using the above Lemma, there is an increasing sequence $\left\{n_{m}\right\}_{m=1}^{\infty}$ of elements of $S$ such that (12) holds with $F=\{\theta\}$. As is well known, the sequence $\{(k+1) \theta$ $(\bmod 2 \pi)\}_{k=1}^{\infty}$ is dense in $[0,2 \pi]$ (cf. e.g. [13, Problem 166, Part II, p. 88]). Hence, for every $\xi \in[0,2 \pi]$ there exist integers $k_{m}$ with $1 \leqslant k_{m} \leqslant m$ such that

$$
\left(k_{m}+1\right) \theta-\arg \psi_{n_{m}}(\theta) \rightarrow \xi \quad(\bmod 2 \pi)
$$

as $m \rightarrow \infty$. Thus (12) and (23) give

$$
\lim _{m \rightarrow \infty} p_{n_{m}+k_{m}}(x)=\frac{t \sin \xi}{y} .
$$

Therefore (22) follows, establishing the Claim.

As an immediate application of this Claim, observe that according to MátéNevai-Totik [6, formula (10.3)] we have

$$
\left.\lim _{n \rightarrow \infty} \int_{-1}^{1}|| \psi_{n}(\theta)\right|^{2} \alpha^{\prime}(x)-\frac{2 y}{\pi} \mid d x=0 .
$$

Since convergence in the mean implies convergence a.e. for a subsequence, we can see that there is an infinite set $S$ of integers such that

$$
\lim _{n \rightarrow \infty, n \in S}\left|\psi_{n}(\theta)\right|=\left(\frac{2 y}{\pi \alpha^{\prime}(x)}\right)^{1 / 2}
$$

holds for almost every $x \in[-1,1]$. Since the restriction that $\theta / 2 \pi$ is irrational excludes only a set of measure zero of the $x$ 's, the above Claim now implies that

$$
\left[-\sqrt{2 / \pi}\left(y \alpha^{\prime}(x)\right)^{-1 / 2}, \sqrt{2 / \pi}\left(y \alpha^{\prime}(x)\right)^{-1 / 2}\right] \subset I(x) \text { a.e. }
$$

This verifies (1), except that we still have to show that, for almost every $x \in[-1,1]$, $I(x)$ is an interval symmetric about the origin.

Writing

$$
b=b(x)=\limsup _{n \rightarrow \infty}\left|p_{n}(x)\right|,
$$

it will be enough to show that

$$
(-b(x), b(x)) \subset I(x)
$$

holds for almost every $x$. In order to do this, assume that $\theta / 2 \pi$ is irrational, and first suppose that $b=b(x)$ is finite. Then, by the definition of $\psi_{n}$ given in (10) (cf. (17) as well), we obtain

$$
\begin{aligned}
b \sin \theta & \leqslant \limsup _{n \rightarrow \infty}\left|p_{n}(x)\right| \sin \theta \leqslant \limsup _{n \rightarrow \infty}\left|\psi_{n}(\theta)\right| \\
& \leqslant \limsup _{n \rightarrow \infty}\left(\left|p_{n}(x)\right|+\left|p_{n-1}(x)\right|\right) \leqslant 2 b .
\end{aligned}
$$

Thus $\left\{\left|\psi_{n}(\theta)\right|\right\}_{n=1}^{\infty}$ has an accumulation point that is at least $b \sin \theta$; hence (23) follows from the Claim. 
Suppose now that $b=b(x)$ is infinite. In addition to assuming that $\theta / 2 \pi$ is irrational, also assume that $x$ is such that (24) holds. Then, by (16) and (17), we have

$$
d(x)=\liminf _{n \rightarrow \infty}\left(\left|p_{n}(x)\right|+\left|p_{n+1}(x)\right|\right)<\infty .
$$

We will show that for every $M>d(x)$ the sequence $\left\{\left|\psi_{n}(\theta)\right|\right\}_{n=1}^{\infty}$ has an accumulation point greater than $M \sin \theta$. From this, (25) will follow in the present case as well by the Claim.

So let $M>d(x)$, and let $S$ be an infinite set of positive integers such that

$$
\left|p_{n}(x)\right|+\left|p_{n+1}(x)\right|<M \quad(n \in S) .
$$

Since $b(x)=+\infty$, for every $n \in S$ there is an integer $n^{\prime}>n+1$ such that $\left|p_{n^{\prime}}(x)\right| \geqslant M$; let $j_{n}$ be the smallest such $n^{\prime}$. Then for large enough $n$ we have

$$
\left|p_{j_{n}}(x)\right| \leqslant 3\left(\left|p_{j_{n}-1}(x)\right|+\left|p_{j_{n}-2}(x)\right|\right) \leqslant 6 M
$$

according to (18). Therefore, using (17), we can see that

$$
(\sin \theta) M \leqslant(\sin \theta)\left|p_{j_{n}}(\theta)\right| \leqslant\left|\psi_{j_{n}}(\theta)\right| \leqslant\left|p_{j_{n}}(x)\right|+\left|p_{j_{n}-1}(x)\right| \leqslant 7 M .
$$

Hence the sequence $\left|\psi_{j_{n}}(\theta)\right|$ has an accumulation point greater than $M \sin \theta$. As remarked above, from this fact (25) follows in the present case as well. The proof of Theorem 1 is complete.

Proof of Corollary. According to Theorem 7.5 in [8, p. 128], (4) implies that $\alpha^{\prime}>0$ a.e. in $[-1,1]$. Hence we can apply Theorem 1 , and (5) follows from (2), completing the proof.

Proof of Theorem 2. Formula (6) was proved as formula (5) in [7, Lemma]. We are going to prove (7) now. The proof is based on formulas (12) and (24). Let $\varepsilon>0$ and $E \subset[-1,1]$ be given as described in the theorem to be proved, and let $\delta>0$ be arbitrary. Using Egoroff's theorem (cf. Halmos [3, Theorem A, p. 80]) twice, we can see that there exists a Lebesgue measurable set $E_{1} \subset E$ with $\left|E \backslash E_{1}\right|<\delta$ such that for $x \in E_{1}$ the convergence in (24) is uniform and such that (12) is valid with $F=E_{1}$, with the error term $o_{\theta}(1)$ tending to zero uniformly for $x \in E_{1}$ ( $x$ and $\theta$ are connected via formula (9); the sets $S$ in (24) and (12) are assumed to be the same). We may assume here that for $x \in E_{1}$ the functions $y=\sqrt{1-x^{2}}$ and $1 / \alpha^{\prime}(x)$ are bounded away from 0 ; then, using (24) in the form stated above, we can see that, for sufficiently large $m,\left|\psi_{n_{m}}(\theta)\right|$ is also uniformly bounded away from zero, provided $x=\cos \theta \in E_{1}$. Let $\varepsilon_{1}$ be arbitrary, with $0<\varepsilon_{1}<\varepsilon$. Using (12) and (24) in the form described, a simple calculation exploiting the boundedness away from zero of the functions mentioned allows us to conclude the relation

$$
\begin{aligned}
\{x \in & \left.E: p_{n_{m}+k}(x)>(1-\varepsilon) \sqrt{2 / \pi}\left(y \alpha^{\prime}(x)\right)^{-1 / 2}\right\} \\
& \supset\left\{x \in E_{1}: \sin \left((k+1) \theta-\arg \psi_{n_{m}}(\theta)\right)>1-\varepsilon_{1}\right\} .
\end{aligned}
$$

Let $\chi_{m, k}$ be the characteristic function of the set on the right side here. For a set $I \subset[0,2 \pi]$ denote by $f(m, I)$ the number of integers $k$ with $1 \leqslant k \leqslant m$ such that $(k+1) \theta \in I(\bmod 2 \pi)$. If $\theta / 2 \pi$ is irrational, then the sequence $\{(k+1) \theta$ $(\bmod 2 \pi)\}_{k=1}^{\infty}$ is equidistributed on $[0,2 \pi]$ (cf. e.g. [13, Problem 166, Part II, p. 88]). 
Thus, for every $I$ that is the union of finitely many intervals we have

$$
\lim _{m \rightarrow \infty} \frac{f(m, I)}{m}=\frac{|I|}{2 \pi} .
$$

In fact, if $I$ runs over sets that can be represented as the union of, say, at most four intervals, then the convergence in (27) is uniform in $I$ (to see this, apply (27) with the intervals $I=[2 \pi j / N, 2 \pi(j+1) N], j=0,1, \ldots, N-1$, for large enough $N)$. Therefore, with fixed $\theta$ such that $\theta / 2 \pi$ is irrational, take $I=I_{m}$ as the mod $2 \pi$-translate by $-\arg \psi_{n_{m}}(\theta)$ of the set

$$
\left\{t \in[0,2 \pi]: \sin t>1-\varepsilon_{1}\right\} \text {. }
$$

Making $m \rightarrow \infty$, the uniformness of the convergence in (27) then implies

$$
\lim _{m \rightarrow \infty} \sum_{k=1}^{m} \frac{\chi_{m, k}(x)}{m}=\frac{1}{\pi} \arccos \left(1-\varepsilon_{1}\right) .
$$

Hence, by the Lebesgue dominated convergence theorem, we have

$$
\lim _{m \rightarrow \infty} \int_{E_{1}} \sum_{k=1}^{m} \frac{\chi_{m, k}(x)}{m} d x=\frac{1}{\pi}\left|E_{1}\right| \arccos \left(1-\varepsilon_{1}\right),
$$

and so, for a sequence $k_{m}$ with $1 \leqslant k_{m} \leqslant m$, we must have

$$
\limsup _{m \rightarrow \infty} \int_{E_{1}} \chi_{m, k_{m}}(x) d x \geqslant \frac{1}{\pi}\left|E_{1}\right| \arccos \left(1-\varepsilon_{1}\right) .
$$

This together with (26) implies

$$
\begin{gathered}
\limsup _{m \rightarrow \infty}\left|\left\{x \in E: p_{n_{m}+k_{m}}(x)>(1-\varepsilon) \sqrt{\frac{2}{\pi}}\left(y \alpha^{\prime}(x)\right)^{-1 / 2}\right\}\right| \\
\geqslant \frac{1}{\pi}\left|E_{1}\right| \arccos \left(1-\varepsilon_{1}\right) .
\end{gathered}
$$

Making $\varepsilon_{1} \rightarrow \varepsilon$ and $\delta \rightarrow 0$ (so that $\left|E_{1}\right| \rightarrow|E|$ ) here, (7) follows. (8) can be established in a similar way. The proof of Theorem 2 is complete.

\section{REFERENCES}

1. G. Freud, Orthogonal polynomials, Pergamon Press, New York, 1971.

2. Ja. L. Geronimus, Orthogonal polynomials, Two Papers on Special Functions, Amer. Math. Soc. Transl. (2) 108 (1977), 37-130. (The Russian original appeared as an appendix added to the Russian Translation of [14], GIFML, Moscow, 1962.)

3. P. R. Halmos, Measure theory, 2nd printing, Springer-Verlag, New York and Berlin, 1974.

4. A. Máté and P. Nevai, Remarks on E. A. Rahmanov's paper "On the asymptotics of the ratio of orthogonal polynomials", J. Approx. Theory 36 (1982), 64-72.

5. A. Máté, P. Nevai and V. Totik, Asymptotics for the ratio of leading coefficients of orthonormal polynomials on the unit circle, Constructive Approx. 1 (1985), 63-69.

6. , Strong and weak convergence of orthogonal polynomials, manuscript.

7. Necessary conditions for the weighted mean convergence of Fourier series in orthogonal polynomials, J. Approx. Theory (to appear).

8. P. Nevai, Orthogonal polynomials, Mem. Amer. Math. Soc. No. 213 (1979).

9. Orthogonal polynomials defined by a recurrence relation, Trans. Amer. Math. Soc. 250 (1979), 369-384.

10. __ On orthogonal polynomials, J. Approx. Theory 25 (1979), 34-37. 
11. E. A. Rahmanov, On the asymptotics of the ratio of orthogonal polynomials, Math. USSR-Sb. 32 (1977), 199-213. (Russian original: Mat. Sb. 103 (1977), 237-252.)

12. __ On the asymptotics of the ratio of orthogonal polynomials. II, Math. USSR-Sb. 46 (1983), 105-117. (Russian original: Mat. Sb. 118 (1982), 104-117.)

13. G. Pólya and G. Szegö, Problems and theorems in analysis. I, Springer-Verlag, New York and Berlin, 1972.

14. G. Szegö, Orthogonal polynomials, 4th ed., Amer. Math. Soc. Colloq. Publ., vol. 23, Amer. Math. Soc., Providence, R. I., 1975.

Department of Mathematics, Brooklyn College, City University of New York, Brooklyn, NEW YORK 11210

Department of Mathematics, Ohio State University, Columbus, Ohio 43210

Bolyai Institute, University of SZeged, 6720 Szeged, Hungary 